USM-TH-149

\title{
A New Approach to Scale Symmetry Breaking and Confinement
}

\author{
P. Gaete ${ }^{1 *}$ and E. I. Guendelman ${ }^{2 \dagger}$ \\ ${ }^{1}$ Departamento de Física, Universidad Técnica F. Santa María, Casilla 110-V, Valparaíso, Chile. \\ ${ }^{2}$ Physics Department, Ben Gurion University, Beer Sheva 84105, Israel.
}

\begin{abstract}
Scale invariant theories which contain (in $4-D$ ) a four index field strength are considered. The integration of the equations of motion of these $4-i n d e x$ field strength gives rise to scale symmetry breaking. The phenomena of mass generation and confinement are possible consequences of this.
\end{abstract}

\section{INTRODUCTION}

The idea that among the fundamental laws of physics, we have scale invariance (s.i.) as one of the fundamental principles appears as a attractive possibility. In its most naive realizations, such a symmetry is not a viable symmetry, however, since nature seems to have chosen some typical scales, so s.i. has to be broken somehow.

While one may look at quantum effects for the origin of scale symmetry breaking, there is also a way to achieve this at the classical level in certain models. In the first scale invariant model of this kind [1], a metric, a dilaton field and a "measure field" $\Phi$ are introduced. The unusual modified measure [2] is an object that has the same transformation properties

\footnotetext{
*E-mail: patricio.gaete@fis.utfsm.cl

$\dagger$ E-mail: guendel@bgumail.bgu.ac.il
} 
under general coordinate transformations as $\sqrt{-g} d^{4} x\left(g=\operatorname{det}\left(g_{\mu \nu}\right)\right) . \Phi$ is a density built out of degrees of freedom independent of the metric. For example, given a four index field strength independent of the metric. for example, given a four index field strength (in four

dimensions) $F_{\mu \nu \alpha \beta}=\partial_{[\mu} A_{\nu \alpha \beta]}$, the measure field $\Phi$ is defined as $\Phi=\varepsilon^{\mu \nu \alpha \beta} F_{\mu \nu \alpha \beta}$. One may consider the field strength to be composed of elementary scalars [3]. Four in the case of a four index field strength, as it was done in Refs. [1,2].

The scale symmetry breaking in Ref. [1] comes when considering the integration of the equation of motion of the four index field strength, which introduces an arbitrary scale in the equations of motion. The modified measure idea has been applied to dynamical generation of string and brane tensions [4], to cosmological [5] and to the fermion family problem [6] and to the cosmic coincidences in cosmology [7].

\section{SPONTANEOUS GENERATION OF CONFINING BEHAVIOR}

Let us now turn our attention to gauge theories and consider first the standard pure Yang-Mills action

$$
S=\int d^{4} x\left(-\frac{1}{4} F_{\mu \nu}^{a} F^{a \mu \nu}\right)
$$

where $F_{\mu \nu}^{a}=\partial_{\mu} A_{\nu}^{a}-\partial_{\nu} A_{\mu}^{a}+g f^{a b c} A_{\mu}^{b} A_{\nu}^{c}$. This theory is invariant under the scale symmetry $A_{\mu}^{a}(x) \rightarrow A_{\mu}^{a}(x)^{\prime}=\lambda A_{\mu}^{a}(\lambda x)$.

Let us rewrite (1) with the help of an auxiliary field $\omega$

$$
S=\int d^{4} x\left(-\frac{1}{4} \omega^{2}+\frac{1}{2} \omega \sqrt{-F_{\mu \nu}^{a} F^{a \mu \nu}}\right)
$$

upon solving the equation of motion obtained from the variation of $\omega$ and then replacing into the action (2) we get back (1).

Let us consider now [8] the replacement $\omega \rightarrow \varepsilon^{\mu \nu \alpha \beta} \partial_{[\mu} A_{\nu \alpha \beta]}$ and consider now the equation of motion obtained from the variation of $A_{\nu \alpha \beta}$, which is

$$
\varepsilon^{\gamma \delta \alpha \beta} \partial_{\beta}\left(\omega-\sqrt{-F_{\mu \nu}^{a} F^{a \mu \nu}}\right)=0
$$


which is solved by

$$
\omega=\sqrt{-F_{\mu \nu}^{a} F^{a \mu \nu}}+M
$$

$M$ being once again a space-time constant which produces s.s.b. of s.i. and in this case it is furthermore associated with the spontaneous generation of confining behavior. Other examples of mass generation were studied also in Ref. [8] with similar technique. Indeed the equations of motion obtained from (2) in the case $\omega$ is replaced by $\varepsilon^{\mu \nu \alpha \beta} \partial_{[\mu} A_{\nu \alpha \beta]}$, have the form

$$
\nabla_{\mu}\left(\left(\sqrt{-F_{\alpha \beta}^{b} F^{b \alpha \beta}}+M\right) \frac{F^{a \mu \nu}}{\sqrt{-F_{\alpha \beta}^{b} F^{b \alpha \beta}}}\right)=0 .
$$

Now study the equation (5) for the case of a Abelian theory and for a spherically symmetric electric field $F_{0 i}=-E_{i}$ and $F_{i j}=0$, where $\mathbf{E}=E(r) \hat{\mathbf{r}}$. Then (5) gives

$$
\nabla \cdot\left(\mathbf{E}+\frac{M}{\sqrt{2}} \hat{\mathbf{r}}\right)=0
$$

which is solved by [9]

$$
\mathbf{E}=-\frac{M}{\sqrt{2}} \hat{\mathbf{r}}+\frac{q}{r^{2}} \hat{\mathbf{r}}
$$

The scalar potential $V$ that gives rise to such electric field is

$$
V=-\frac{M}{\sqrt{2}} r+\frac{q}{r}
$$

which is indeed resembles very much the Cornell confining potential. Notice that so far (8) refers to the field of one charge and not yet to the interaction energy between two charges. We will see that such interaction energy also has the Cornell form, even at the quantum level. Since Abelian solutions are solutions of the non-Abelian theory, these solutions are also relevant for the non-Abelian generalization.

Before approaching the quantum theory (which will be treated in some approximations) we want to define effective actions that give the equations of motion (5). Indeed one can easily see that [9] 


$$
L_{e f f}=-\frac{1}{4} F_{\mu \nu} F^{\mu \nu}-\frac{M}{4} \sqrt{-F_{\mu \nu} F^{\mu \nu}}
$$

reproduces Eqs. (5).

Since the full treatment of the quantum theory is rather difficult, instead of using (9) we restrict ourselves to a "truncated" phase space model where we consider spherical coordinates $(r, \theta, \varphi)$ in addition to time, but where we set $F_{i j}=0=F_{0 \varphi}=F_{0 \theta}$ and consider only $(t, r)$ dependence of $F_{0 r}$. Then instead of (9), we consider

$$
S=4 \pi \int d r r^{2} L_{e f f}
$$

where

$$
L_{e f f}=\frac{1}{2}\left(F_{0 r}\right)^{2}-\frac{M \sqrt{2}}{4} F_{0 r}
$$

Similar kind of "reduced phase space" which take into account only the spherical degrees of freedom have been used elsewhere in other examples, see for example Ref. [10].

\section{INTERACTION ENERGY}

As already mentioned, our aim now is to calculate the interaction energy between external probe sources in the model (10). To do this, we will compute the expectation value of the energy operator $H$ in the physical state $|\Phi\rangle$, which we will denote by $\langle H\rangle_{\Phi}$. The starting point is the two-dimensional space-time Lagrangian (11):

$$
L=4 \pi r^{2}\left\{-\frac{1}{4} F_{\mu \nu} F^{\mu \nu}-\frac{M \sqrt{2}}{8} \varepsilon_{\mu \nu} F^{\mu \nu}\right\}-A_{0} J^{0},
$$

where $J^{0}$ is the external current. A notation remark, in (12), $\mu, \nu=0,1$, also, $x^{1} \equiv r \equiv x$ and $\varepsilon^{01}=1$.

We now proceed to obtain the Hamiltonian. For this we restrict our attention to the Hamiltonian framework of this theory. The canonical momenta read $\Pi^{\mu}=$ $-4 \pi x^{2}\left(F^{0 \mu}+\frac{M \sqrt{2}}{8} \varepsilon^{0 \mu}\right)$, which results in the usual primary constraint $\Pi^{0}=0$, and $\Pi^{i}=$

$-4 \pi x^{2}\left(F^{0 i}+\frac{M \sqrt{2}}{8} \varepsilon^{0 i}\right)$. The canonical Hamiltonian following from the above Lagrangian is: 


$$
H_{C}=\int d x\left(\Pi_{1} \partial^{1} A^{0}-\frac{1}{8 \pi x^{2}} \Pi_{1} \Pi^{1}-\frac{M \sqrt{2}}{4} \varepsilon^{01} \Pi_{1}+A_{0} J^{0}\right) .
$$

The consistency condition $\dot{\Pi}_{0}=0$ leads to the secondary constraint $\Gamma_{1}(x) \equiv \partial_{1} \Pi^{1}-J^{0}=0$. It is straightforward to check that there are no further constraints in the theory, and that the above constraints are first class. The extended Hamiltonian that generates translations in time then reads $H=H_{C}+\int d x\left(c_{0}(x) \Pi_{0}(x)+c_{1}(x) \Gamma_{1}(x)\right)$, where $c_{0}(x)$ and $c_{1}(x)$ are the Lagrange multipliers. Moreover, it follows from this Hamiltonian that $\dot{A}_{0}(x)=\left[A_{0}(x), H\right]=$ $c_{0}(x)$, which is an arbitrary function. Since $\Pi_{0}=0$, neither $A^{0}$ nor $\Pi^{0}$ are of interest in describing the system and may be discarded from the theory. The Hamiltonian then takes the form

$$
H=\int d x\left(-\frac{1}{8 \pi x^{2}} \Pi_{1} \Pi^{1}-\frac{M \sqrt{2}}{4} \varepsilon^{01} \Pi_{1}+c^{\prime}\left(\partial_{1} \Pi^{1}-J^{0}\right)\right),
$$

where $c^{\prime}(x)=c_{1}(x)-A_{0}(x)$.

According to the usual procedure we introduce a supplementary condition on the vector potential such that the full set of constraints becomes second class. A convenient choice is found to be [11-14]

$$
\Gamma_{2}(x) \equiv \int_{C_{\xi x}} d z^{\nu} A_{\nu}(z) \equiv \int_{0}^{1} d \lambda x^{1} A_{1}(\lambda x)=0,
$$

where $\lambda(0 \leq \lambda \leq 1)$ is the parameter describing the spacelike straight path $x^{1}=\xi^{1}+$ $\lambda(x-\xi)^{1}$, and $\xi$ is a fixed point (reference point). There is no essential loss of generality if we restrict our considerations to $\xi^{1}=0$. In this case, the only nontrivial Dirac bracket is

$$
\left\{A_{1}(x), \Pi^{1}(y)\right\}^{*}=\delta^{(1)}(x-y)-\partial_{1}^{x} \int_{0}^{1} d \lambda x^{1} \delta^{(1)}(\lambda x-y) .
$$

We are now equipped to compute the interaction energy between pointlike sources in the model (14), where a fermion is localized at the origin $\mathbf{0}$ and an antifermion at $\mathbf{y}$. As we have already mentioned, we will calculate the expectation value of the energy operator $H$ in the physical state $|\Phi\rangle$. From our above discussion, we see that $\langle H\rangle_{\Phi}$ reads

$$
\langle H\rangle_{\Phi}=\left\langle\Phi\left|\int d x\left(-\frac{1}{8 \pi x^{2}} \Pi_{1} \Pi^{1}-\frac{M \sqrt{2}}{4} \varepsilon^{01} \Pi_{1}\right)\right| \Phi\right\rangle .
$$


Next, as remarked by Dirac [15], the physical state can be written as

$$
|\Phi\rangle \equiv|\bar{\Psi}(\mathbf{y}) \Psi(\mathbf{0})\rangle=\bar{\psi}(\mathbf{y}) \exp \left(i e \int_{\mathbf{0}}^{\mathbf{y}} d z^{i} A_{i}(z)\right) \psi(\mathbf{0})|0\rangle,
$$

where $|0\rangle$ is the physical vacuum state. As we have already indicated, the line integral appearing in the above expression is along a spacelike path starting at $\mathbf{0}$ and ending $\mathbf{y}$, on a fixed time slice.

Taking into account the above Hamiltonian structure, we observe that

$$
\Pi_{1}(x)|\bar{\Psi}(y) \Psi(0)\rangle=\bar{\Psi}(y) \Psi(0) \Pi_{1}(x)|0\rangle-e \int_{0}^{y} d z_{1} \delta^{(1)}\left(z_{1}-x\right)|\Phi\rangle .
$$

Inserting this back into (17), we get

$$
\langle H\rangle_{\Phi}=\langle H\rangle_{0}+\frac{e^{2}}{8 \pi} \int d x \frac{1}{x^{2}}\left(\int_{0}^{y} d z_{1} \delta^{(1)}\left(z_{1}-x\right)\right)^{2}+\frac{M \sqrt{2} e}{4} \int d x\left(\int_{0}^{y} d z_{1} \delta^{(1)}\left(z_{1}-x\right)\right),
$$

where $\langle H\rangle_{0}=\langle 0|H| 0\rangle$. We further note that

$$
\frac{e^{2}}{2} \int d x\left(\int_{0}^{y} d z \delta^{(1)}\left(z_{1}-x\right)\right)^{2}=\frac{e^{2}}{2} L,
$$

with $|y| \equiv L$. Inserting this into Eq.(20), the interaction energy in the presence of the static charges will be given by

$$
V=-\frac{e^{2}}{8 \pi} \frac{1}{L}+\frac{M \sqrt{2} e}{4} L,
$$

which has the Cornell form. In this way the static interaction between fermions arises only because of the requirement that the $|\bar{\Psi} \Psi\rangle$ states be gauge invariant. 


\section{REFERENCES}

[1] E. I. Guendelman, Mod. Phys. Lett. A14, 1043 (1999); Mod. Phys. Lett. A14, 1397 (1999); gr-qc/9901067.

[2] For a review on non scale invariant versions of these models see E. I. Guendelman and A. B. Kaganovich, Phys. Rev. D60, 065004 (1999). Early papers on the subject: E. I. Guendelman Phys. Rev. D53, 7020 (1996); Phys. Rev. D55, 5970 (1997).

[3] E. I. Guendelman, E. Nissimov and S. Pacheva, Phys. Lett. B360, 57 (1995); hepth/9903245; C. Castro Int. J. Mod. Phys. A13, 1263 (1998).

[4] E. I. Guendelman, Class Quantum Grav. 17, 3673 (2000); E. I. Guendelman, Phys. Rev. D63, 046006 (2001); E. I. Guendelman, A. B. Kaganovich, E. Nissimov and S. Pacheva, Phys. Rev. D66, 046003 (2002); hep-th/0304269; hep-th/0210062.

[5] E. I. Guendelman and O. Katz, Class. Quantum Grav 20, 1715 (2003) and references there.

[6] E. I. Guendelman and A. B. Kaganovich, Int. J. Mod. Phys. A17, 417 (2002); Mod. Phys. Lett. A17, 1227 (2002); Int. J. Mod. Phys. A17, 4419 (2002); Int. J. Mod. Phys. D11, 1595 (2002).

[7] E. I. Guendelman and A. B. Kaganovich, gr-qc/0312006.

[8] E. I. Guendelman, hep-th/0306162.

[9] P. Gaete and E. I. Guendelman, hep-th/0308025.

[10] R. Benguria, P. Cordero and C. Teitelboim, Nucl.Phys. B122, 61 (1977).

[11] P. Gaete, Phys. Lett. B515, 32 (2001).

[12] P. Gaete, Phys. Rev. D59, 127702 (1999).

[13] P. Gaete and I. Schmidt, Phys. Rev. D61, 125002 (2000). 
[14] P. Gaete and I. Schmidt, Phys. Rev. D64, 027702 (2001).

[15] P. A. M. Dirac, The Principles of Quantum Mechanics (Oxford University Press, Oxford, 1958); Can. J. Phys. 33, 650 (1955). 